\title{
A High Incidence of Native Portal Vein Thrombosis in Veterans Undergoing Liver Transplantation
}

\author{
Timothy J. Gayowski, M.D., Ignazio R. Marino, M.D., Howard R. Doyle, M.D., Luis EcheverRi, M.D., \\ Luis Mieles, M.D., SATORU Todo, M.D., Marilyn Wagener, M.P.H., Nina Singh, M.D., \\ Victor L. Yu, M.D., John J. Fung, M.D., Ph.D., And Thomas E. STarZl, M.D., Ph.D. \\ Veterans Administration Medical Center and the Pittsburgh Transplantation Institute, University of Pittsburgh, \\ Pittsburgh, Pennsylvania 15213
}

Presented at the Annual Symposium of the Association of Veterans Administration Surgeons, Pittsburgh, Pennsylvania, May 1-3, 1995

The incidence of native portal vein thrombosis (PVT) in liver transplant recipients has been reported to range from 2.1 to $13.8 \%$. We have identified an inordinately high incidence of PVT in a consecutive series of U.S. veterans receiving liver transplants. Between October 1989 and February 1994, 88 consecutive U.S. veterans received 99 orthotopic liver transplants under primary Tacrolimus (Prograf, formerly FK506) based immunosuppression. A number of clinical features were examined in an effort to identify risk factors for PVT and outcome was compared to patients without PVT. Native PVT was present in 23/88 (26\%) patients. All of these patients were male U.S. veterans with a mean age of 47 years. When compared to the 65 patients without PVT, we found no significant difference with respect to underlying liver disease, age, Childs-Pugh score (mean $=12)$, UNOS status as defined prior to April 1995 (95\% UNOS 3 or 4), previous abdominal surgery, or liver volume. Median blood loss for patients with PVT (21 units of packed red blood cells) was greater than for those without PVT (14 units, $P=0.04)$. Portal thrombectomy was performed in 11 patients, 11 patients required mesoportal jump grafts, and 1 patient had an interposition graft. Standard veno-venous bypass was used in 10 patients with single bypass utilized for the remainder. Actuarial patient survival for all patients at 1,2 , and 4 years was 88,85 , and $79 \%$, respectively. There was no significant difference in patients with or without PVT. Patients with PVT had poorer graft survival than patients without PVT (86\% vs $65 \%$, 1 year; $81 \%$ vs $65 \%$, 2 years; $81 \%$ vs $61 \%, 4$ years; $P=0.03)$; however, this was not related to technical problems with the portal venous inflow. PVT occurred in $26 \%$ of U.S. veterans undergoing liver transplantation. These patients had significantly higher operative blood loss and poorer graft survival. The high incidence of postnecrotic cirrhosis in a predominantly male group of patients with advanced disease, as is evident by the high mean Childs-Pugh score and UNOS status, perhaps accounts for our observations. o) 1996 Academic Press, Inc.

\section{INTRODUCTION}

Portal vein thrombosis (PVT) and other permutations of splanchnic venous thromboses, are no longer considered relative contraindications to orthotopic liver transplantation (OLTX) [1-5]. Historically, the incidence of portal vein thrombosis in cirrhotics has been reported to be $11 \%[6]$. It is reported to be more common in male patients as well as in patients with BuddChiari syndrome and postnecrotic cirrhosis. More recent studies indicate that the incidence in cirrhosis ranges from 0.5 to $21 \%[6-9]$.

The incidence of native portal vein thrombosis in liver transplant recipients has been reported to range from 2.1 to $13.8 \%[1-5]$. Male patients, patients with Laennec's cirrhosis, chronic active viral hepatitis, Budd-Chiari syndrome, previous portosystemic shunt, hypercoagulable states, trauma, previous portahepatis dissection, or postsplenectomy appear to be at a higher risk for PVT [1-5, 10-17]. Demographic information regarding patients without portal vein thrombosis in these series are often lacking and, hence, data regarding other potential risk factors are sparse. A report from the University of Pittsburgh identified patients with encephalopathy, ascites, variceal bleeding, previous splenectomy, and a small liver as having a higher incidence of PVT [4].

Under primary Tacrolimus (Prograf, formerly FK506) based immunosuppression, we have identified a high incidence of native PVT in U.S. veterans undergoing OLTX. We have examined a number of clinical features in this consecutive series of patients to try and identify risk factors for PVT and ascertain the reason for such a high incidence in our select patient population. Our reconstructive approach for portal revascularization is also described.

\section{MATERIALS AND METHODS}

Patient material. At the Veterans Administration Medical Center in Pittsburgh, 99 OLTX were performed in 88 patients ( 87 males, 1 female) under Tacrolimus (Prograf, formerly FK506) based immunosuppression between October 1, 1989, and February 28, 1994. Data 
was obtained from medical records review and from a prospective data base for veterans undergoing primary liver transplantation under Tacrolimus. The immunosuppressive regimen has been described previously and the patient's demographics were similar to those in the earlier reported series [18]. In this veteran population, $23 \mathrm{pa}$ tients (26\%) were found to have PVT. All patients were male with a mean age of 46.7 years. Thirteen patients had postnecrotic cirrhosis secondary to viral hepatitis (hepatitis C [13], hepatitis B and C [2]). Six patients had alcoholic cirrhosis and 6 of the aforementioned patients with viral hepatitis had a history of alcohol abuse. The remaining indications for liver transplantation included sclerosing cholangitis [2], Wilson disease [1] and alpha-I antitrypsin deficiency [1]. Four patients had hepatocellular carcinomas (alcoholic cirrhosis [3], Wilson disease [1]) of which two were incidental $(<2 \mathrm{~cm})$. Portal vein thrombi were benign in all four patients. The extent of extrahepatic portosplanchnic venous thrombosis was classified by the following grading system: (a) Grade 1, a partial(mural) thrombosis of the main portal trunk extending to or below the confluence with residual flow; (b) Grade 2, complete thrombosis of the main portal trunk not extending to the confluence of the superior mesenteric and splenic veins; (c) Grade 3, complete thrombosis of the main portal trunk extending to the level of the confluence; (d) Grade 4, complete thrombosis of the main portal trunk with extension below the confluence.

Patency of the portal vein was assessed by Doppler ultrasonography in all cases. Absence of flow or poor flow was further investigated using selective celiac and mesenteric angiography or magnetic resonance imaging if renal function was impaired. Post-transplantation evaluation of portal venous inflow was carried out by routine early ( $<7$ days) and yearly doppler ultrasonography. Autopsy findings in early and late patient deaths were used to determine the patency of portal inflow.

The following clinical parameters were examined as possible risk factors for native PVT in liver transplant recipients: etiology of liver disease, age, Childs-Pugh score, previous abdominal surgery, UNOS status, liver volume (size), presence of ascites, a history of spontaneous bacterial peritonitis (SBP), encephalopathy, variceal bleeding, and endoscopic sclerotherapy for variceal bleeding. The impact of native PVT on patient survival, graft survival, retransplantation rate, transfusion requirement, and postoperative infectious morbidity (major bacterial, fungal, and viral) was also examined.

Statistical analysis. Clinical and laboratory values were entered into a database (PROPHET Statistics, BBN Systems and Technologies, Cambridge MA). Categorical variables (mortality, infection) were compared using the $\chi^{2}$ test or the Fisher exact test. Ordinal values such as UNOS or Childs-Pugh score were compared using the Mann-Whitney test. Kaplan-Meier survival curves were constructed using the date of first transplant as the starting point and loss of graft or death as the end points. Survival curves were compared using the Mantel-Cox long-rank test.

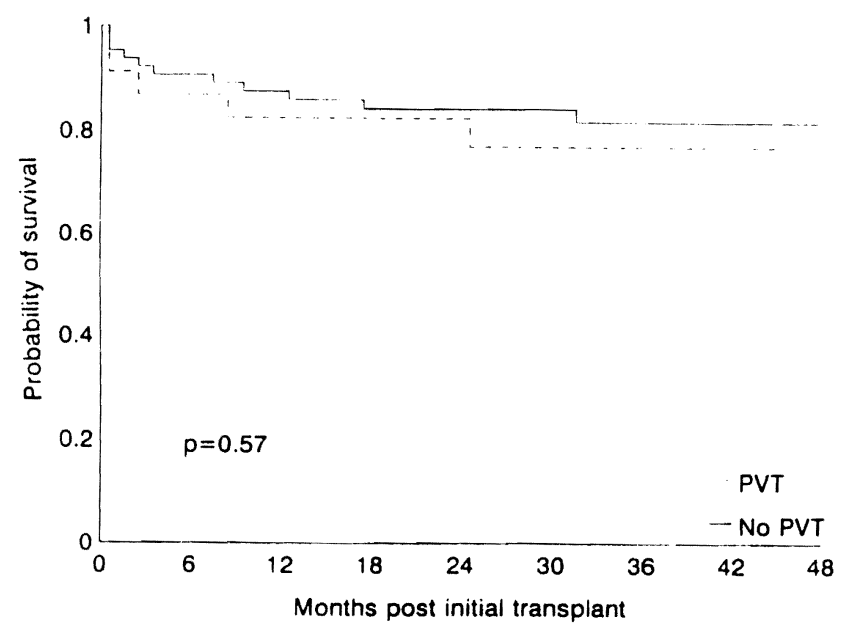

FIG. 1. Actuarial (Kaplan-Meier) patient survival after liver transplantation in patients with and without PVT.

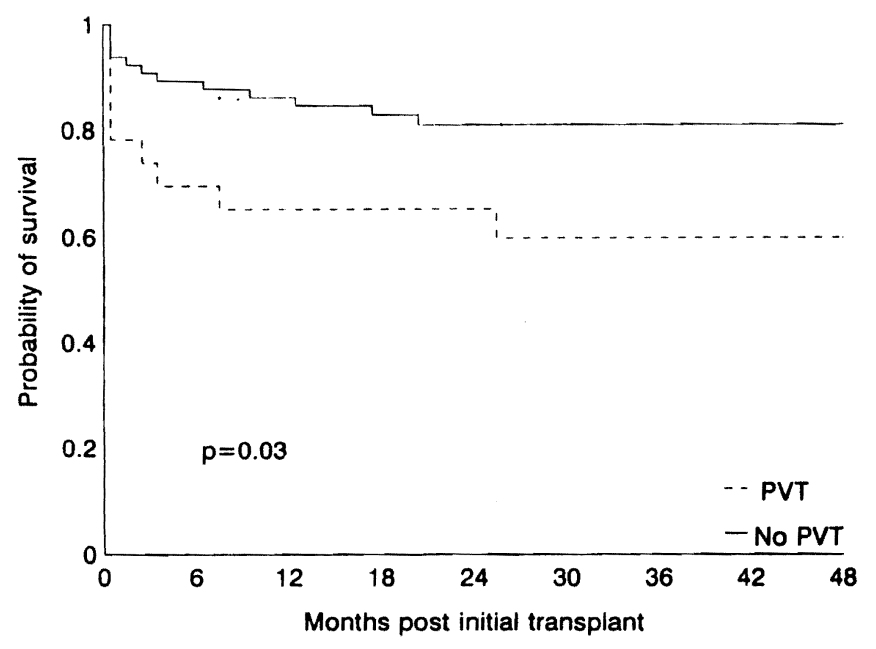

FIG. 2. Actuarial (Kaplan-Meier) graft survival after liver transplantation in patients with and without PVT.

\section{RESULTS}

The actuarial survival rates for all 88 patients after liver transplantation under primary 'Tacrolimus-based immunosuppression are 88,85 , and $79 \%$ at 1,2 , and 4 years, respectively.

Patients have been followed for a mean of 38.7 months (median, 41.5 months; range, 13 to 64 months). Patient survival did not differ significantly in patients with or without PVT ( $83 \%$ vs $88 \%, 1$ year; $83 \%$ vs $84 \%$, 2 years; $77 \%$ vs $81 \%$, 4 years) (Fig. 1 ). Patients with PVT had poorer graft survival with a larger number of early graft losses (Fig. 2) ( $85 \%$ vs $65 \%, 1$ year; $81 \%$ vs $65 \%, 2$ years; $81 \%$ vs $61 \%, 4$ years; $P=0.03$ ). These graft losses were not a consequence of technical problems related to portal venous inflow.

Eight patients $(39 \%)$ had undergone previous intraabdominal surgery. Procedures included an exploratory laparotomy for trauma, distal splenorenal shunt, total colectomy, cholecystectomy [3], and appendectomy $|2|$. All patients had Childs-Pugh class C liver disease (mean score 12.4). Ten patients were in the intensive care unit (UNOS status 4) before transplantation and the remaining 13 patients were hospitalized (UNOS 3). Liver size and age were similar in those with and without PVT. Characteristics of the patients with and without PVT are shown in Table 1.

\section{Complications of Portal Hypertension and Portal Vein Thrombosis}

The association of various consequences of portal hypertension (ascites, spontaneous bacterial peritonitis, encephalopathy, variceal bleeding \pm sclerotherapy) in patients with or without PVT was examined (Table 2). No statistically significant differences between the two groups were observed.

\section{Radiologic Studies}

Routine pretransplant Doppler ultrasonography showed a patent portal venous system in 17 of 23 pa- 
TABLE 1

Risk Factors for Native Portal Vein Thrombosis

\begin{tabular}{|c|c|c|c|}
\hline Risk factor & PVT $(n=23)$ & No PVT $(n=65)$ & $P$ value \\
\hline \multicolumn{4}{|l|}{ Diagnosis } \\
\hline \multicolumn{4}{|l|}{$\begin{array}{r}\text { Postnecrotic } \\
\text { cirrhosis }\end{array}$} \\
\hline Alcohol & $54 \%$ & $42 \%$ & NS \\
\hline Hepatitis C & $57 \%$ & $48 \%$ & \\
\hline Hepatitis B & $8 \%$ & $9 \%$ & \\
\hline \multicolumn{4}{|l|}{ Alcohol and } \\
\hline hepatitis $^{a}$ & $26 \%$ & $20 \%$ & \\
\hline \multicolumn{4}{|l|}{ Primary biliary } \\
\hline cirrhosis & 0 & $3 \%$ & \\
\hline \multicolumn{4}{|l|}{ Sclerosing } \\
\hline \multirow{2}{*}{\multicolumn{4}{|c|}{$\begin{array}{l}\text { Metabolic } \\
\text { a-I antitrypsin }\end{array}$}} \\
\hline & & 0 & \\
\hline Wilson disease & $\begin{array}{l}4 \% \\
4 \%\end{array}$ & $\begin{array}{l}0 \\
0\end{array}$ & \\
\hline \multicolumn{4}{|l|}{ Hepatocellular } \\
\hline carcinoma & $17 \%$ & $6 \%$ & \\
\hline Age (mean) & 46 & 47 & NS \\
\hline \multicolumn{4}{|l|}{ Sex } \\
\hline Male & 23 & 64 & NS \\
\hline Female & 0 & 1 & \\
\hline \multicolumn{4}{|l|}{ Previous } \\
\hline Yes & $39 \%$ & $38 \%$ & NS \\
\hline \multicolumn{4}{|l|}{ UNOS status } \\
\hline 2 & 0 & $4 \%$ & NS \\
\hline 3 & $57 \%$ & $63 \%$ & \\
\hline 4 & $43 \%$ & $32 \%$ & \\
\hline \multicolumn{4}{|l|}{ Liver volume } \\
\hline Mean & 1619 & 1684 & \\
\hline Range & $749-3072$ & $888-3315$ & \\
\hline \multicolumn{4}{|l|}{ Childs-Pugh } \\
\hline score (mean) & 12 & 12 & NS \\
\hline
\end{tabular}

${ }^{a}$ Patients with both a history of alcohol use and viral hepatitis (6/ 24 with PVT, $13 / 65$ without PVT).

tients. Six of these patients had incomplete thrombosis (Grade 1) with residual flow discovered at surgery or with angiography [2], while the remainder had complete obstruction ( 11 false negative studies). We routinely perform pretransplant angiography when ultrasonography fails to demonstrate portal flow or there is questionable (low) flow. In cases where hepatofugal flow is demonstrated, angiography is, in general, reserved for those with low flow. Selective celiac and mesenteric angiography documented complete obstruction in 6 patients, while two studies showed small patent portal veins with mural thrombosis and spontaneous portosystemic shunts. Six intraoperative portograms were carried out to define better the splanchnic venous return for reconstruction. Magnetic resonance imaging studies indicated the portal venous system to be falsely patent in 2 patients, while one study indicated complete thrombosis in a patient with marginal flow shown by ultrasonography ( $2 / 3$ false negative studies).

\section{Surgical Approach}

The extent of portal-splanchnic thrombosis and reconstructive approach for this series of patients is shown in Table 3. The importance of routine procurement of iliac vessels was established in the early days of liver transplantation [19]. Procurement and vein grafting techniques have been well described and these were applied in this case series $[10-15,20]$. Intraoperative assessment with dissection of the portal vein to the confluence of the superior mesenteric and splenic veins revealed incomplete thrombosis (Grade 1) of the main portal trunk extending to or below the confluence in 6 cases. Thrombectomy was carried out in all 6 cases with end-end anastomotic reconstruction. In 4 patients with complete thrombosis not extending to the confluence (Grade 2), thrombectomy was also utilized with end-end reconstruction. Extension to or below the confluence with complete thrombosis (Grades 3 and 4) was noted in the remainder with reconstruction using allogeneic iliac vein mesoportal jump grafts (transmesocolic) (Fig. 3) in 11 patients, thrombectomy with end to end anastomosis in 1 patient, and an interposition graft to the confluence in 1 . Two patients had spontaneous splenorenal shunts, however, portal flow via the iliac vein grafts was excellent and ligation of the shunt and collaterals was not necessary. Post-transplant allograft function was excellent in both patients. Transplantation in patients with PVT was associated with a significantly greater blood loss with a median use of 21 units of packed red blood cells versus 14 units in patients without PVT $(P=0.04)$. Standard venovenous bypass was used in 10 patients (after thrombectomy) with single bypass utilized in the remainder.

\section{Mortality, Morbidity, and Retransplantation}

There were five patient deaths (21\%). Operative deaths (within the first 30 days) occurred in two patients and 1 patient died at 70 days. One operative death was the result of severe coagulopathy, massive transfusion, and myocardial failure with accompanying intractable dysrhythmias postoperatively. The other patient experienced primary graft nonfunction in the first two allografts with documented heavy deposition of complement and immunoglobulin consistent with hyperacute rejection. This patient expired after a third attempt at transplantation with a myocardial infarction followed by multiple systems organ failure.

\section{TABLE 2} Incidence of Portal Vein Thrombosis and
Complications of Portal Hypertension

\begin{tabular}{lccc}
\hline \multicolumn{1}{c}{ Condition } & PVT $(n=23)$ & No PVT $(n=65)$ & $P$ value \\
\hline $\begin{array}{l}\text { Encephalopathy } \\
\text { (Grades 2-4) }\end{array}$ & $11(48 \%)$ & $27(41 \%)$ & 0.6 \\
$\begin{array}{l}\text { Ascites } \\
\begin{array}{l}\text { Spontaneous } \\
\text { bacterial }\end{array}\end{array}$ & $17(74 \%)$ & $40(61 \%)$ & 0.3 \\
$\quad$ peritonitis & $5(22 \%)$ & $23(35 \%)$ & 0.2 \\
$\begin{array}{l}\text { Variceal bleed } \\
\text { Sclerotherapy/ }\end{array} \quad 5(22 \%)$ & $15(23 \%)$ & 0.9 \\
$\quad$ variceal bleed & $4(17 \%)$ & $6(9 \%)$ & 0.3 \\
\hline
\end{tabular}


TABLE 3

Extent of Portal-Splanchnic Thrombosis and Method of Reconstruction

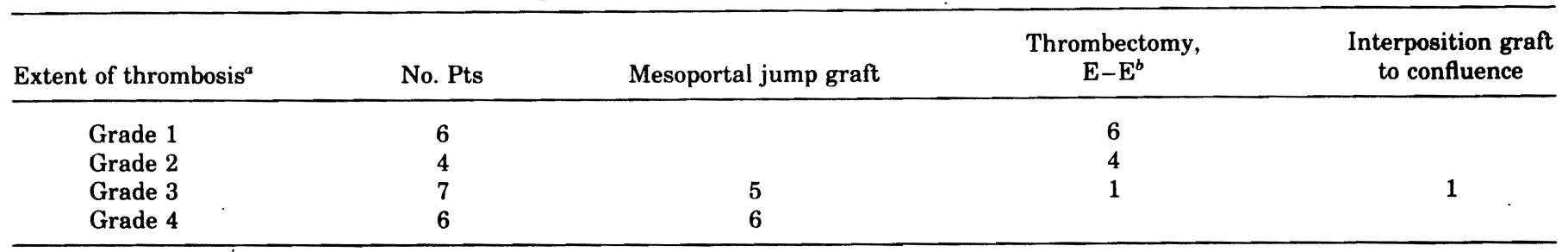

${ }^{a}$ Grade 1, mural thrombosis of main portal trunk ( \pm extension below) with residual flow; Grade 2, complete thrombosis of the main portal trunk not extending to the confluence; Grade 3, complete thrombosis of the main portal trunk extending to the confluence; Grade 4, complete thrombosis of the main portal trunk with extension below the confluence.

${ }^{b}$ End-end anastomosis.

The patient death at 70 days was the result of a family decision to withdraw support. One late patient death at 26 months was the result of allograft failure secondary to recurrent hepatitis $B$ (DNA positive). A second late death (9 months) from sepsis occurred after retransplantation. Patent portal venous inflow was documented in all five patients at autopsy.

Dialysis-dependent renal failure was seen in five patients. Two patients required long-standing hemodialysis ( $>1$ month) pretransplant with renal failure resolving postoperatively in one. Hemodialysis was temporary in the remainder. Exploratory laparotomy for bleeding was required in one patient. Two patients had clinical peritonitis warranting exploration with primary peritonitis found in one patient and secondary

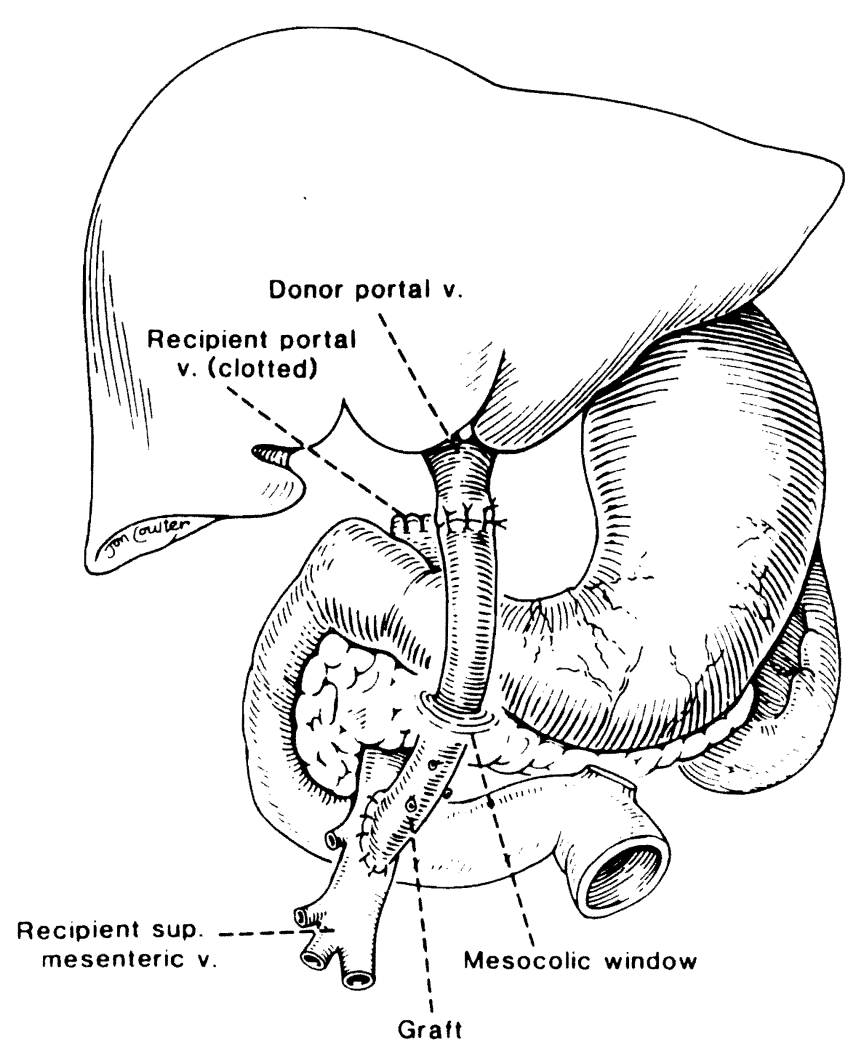

FIG. 3. Transmesocolic allogeneic iliac vein meso-portal jump graft. peritonitis due to patchy areas of necrosis found in the small intestine of the other. Biliary complications included a small contained bile leak from a choledochojejunostomy and biliary cast formation both requiring reconstruction. This surgical morbidity was unrelated to portal venous inflow or vascular anastomotic complications. The patient with biliary cast formation died with recurrent hepatitis B (see above) and the remainder are all alive and well. Post-transplant surgical morbidity was similar in patients with and without PVT.

The mean number of major infections (bacterial or fungal) in the PVT group did not differ significantly from those without PVT. The incidence of CMV disease was similar in both groups at $17 \%$ in patients with PVT and $12 \%$ in those without (Table 4).

Retransplantation was necessary in six patients (26\%). Four received a second allograft, while one patient received three grafts. Early retransplantation ( $<7$ days) was performed for primary nonfunction in four patients with satisfactory function of the new allograft in three patients. The fourth patient in this early retransplant group experienced repeated graft nonfunction with evidence of hyperacute rejection. A fifth patient was retransplanted at 95 days because of significant arterial stenosis at the level of the bifurcation of the right and left hepatic arteries. Reconstruction of the arterial inflow to the first allograft was necessary at this high level because of an intimal dissection in the donor artery. The sixth patient required retransplantation at 8.5 months for chronic rejection and died of staphylococcal sepsis and anoxic brain injury 29 days after retransplantation with normal graft function.

TABLE 4

Infectious Morbidity after OLTx and Native PVT

\begin{tabular}{lccl}
\hline \multicolumn{1}{c}{ Infection } & PVT $(n=23)$ & No PVT $(n=65)$ & $P$ value \\
\hline Viral & & & \\
CMV shedding & $9(39 \%)$ & $20(31 \%)$ & 0.4 \\
CMV disease & $4(17 \%)$ & $8(12 \%)$ & 0.5 \\
Major bacterial $^{a}$ & $10(43 \%)$ & $18(28 \%)$ & 0.16 \\
Fungal (invasive) & $1(4 \%)$ & $7(11 \%)$ & 0.4 \\
\hline
\end{tabular}

a Defined as bacteremia, intraabdominal abscess, wound infection, peritonitis, c. difficile colitis, cholangitis. 
Only four patients $(6 \%)$ required retransplantation in the non-PVT group $(P=0.02)$.

\section{DISCUSSION}

As a result of refinements in techniques and experience with complex portal venous reconstruction, the presence of thrombus in the portal vein and its tributaries is not a contraindication for liver transplantation [1-5]. Nonetheless, few would argue that these patients represent a greater technical challenge.

We know historically that mortality is ensured should we fail to establish portal venous inflow in the transplanted liver [21]. It is mandatory, therefore, to document patency of the portal system prior to transplantation. Like most centers, we rely.on duplex ultrasonography which is a simple, noninvasive technique [22-24]. Although others have reported a high degree of sensitivity for PVT with duplex scans, we have not had the same experience [1]. As a result, our threshold for pursuing further imaging studies such as selective splanchnic angiography has lessened. We reserve the use of intraoperative portography for cases of unexpected complete (Grades 2-4) PVT in which the anatomy is not clear.

It has been observed that PVT appears to be more common in males and in patients with Laennec's cirrhosis or chronic active hepatitis $(\mathrm{CAH})[1,2,17]$. We did not find any statistically significant difference between our patients with and without PVT and their underlying etiology of liver disease. It is notable, however, that we have a very high incidence of postnecrotic cirrhosis (Laennec's +/- CAH)(84\% in patients with PVT, $86 \%$ in patients without PVT). This is perhaps one factor that may account for the high overall incidence of PVT in our veteran population. It has been hypothesized that the higher rate of PVT in patients with postnecrotic cirrhosis may be attributable to a higher impedance of flow through what are generally smaller, shrunken livers. Although a previous report found liver volume (size) to be an important risk factor [4], we did not find any significant difference in liver volume between the two groups. Consistent with the higher impedance to flow hypothesis is our observation of severe portal hypertension in the vast majority of our patients with PVT (only two had spontaneous portosystemic shunts with decompression).

Although we had a relatively high incidence of previous abdominal surgery in both groups, we did not have an appreciable number of patients with previous splenectomy or portosystemic shunt surgery to allow for meaningful statistical analysis of these specific risk factors previously alluded to $[2,4,7]$. Previous upper abdominal surgery certainly added to the technical challenge in our patients with PVT and not unexpectedly the mean transfusion requirement was significantly higher in this group $(P=0.04)$.

Other risk factors for PVT that reflect severity of disease were also examined. We did not find any difference in the two groups with respect to UNOS status
( $>95 \%$ status $3-4)$, Childs-Pugh score (mean $=12$ in both), or complications of portal hypertension including consequences of endoscopic variceal sclerotherapy in contrast to the findings of Nonami et al. and others [4, 17]. The incidence of encephalopathy, ascites, and SBP was higher overall in our series of patients and these factors coupled with a high mean Childs-Pugh score and UNOS status simply reflect a high risk group of patients overall with advanced disease.

Post-transplantation infectious morbidity was examined in both groups with expectations that major bacterial or invasive fungal infections were more prevalent in patients with PVT [2]. With the higher retransplantation rate in our patients with PVT, a higher rate of infections, complications, and mortality are expected $[25,26]$. We did not observe any statistically significant differences in viral, major bacterial, or fungal infections between the two groups in our series, although bacterial infections were observed more frequently in patients with PVT $(P=0.16)$. This is surprising but, nevertheless, consistent with our previously published observations in which our overall incidence of major bacterial infections was only $39 \%$ (27).

Our surgical approach to patients with PVT is dependent upon the determination of the extent of splanchnic venous thrombosis and the degree of portal hypertension and collaterization in the portahepatis and peripancreatic region. We concur with Stieber et al. (1) and Langnas et al. (2) and try to avoid extensive peripancreatic dissection, especially in patients with Grades 3-4 PVT. We have a low threshold to employ transmesocolic mesoportal jump grafts, and we believe that this has resulted in less morbidity (no cases of pancreatitis) in patients with advanced portal-splanchnic thrombosis. With nonocclusive disease or less extensive occlusive (Grade 2) PVT, we prefer thrombectomy with end to end reconstruction or the use of an interposition graft if necessary.

The overall actuarial 1-, 2-, and 4-year survival rates were 88,85 , and $79 \%$, respectively. Survival in patients with PVT were slightly less than those without at 1 year (83\% with PVT, $88 \%$ no PVT); however, this was not statistically significant. Patients with PVT did have significantly diminished graft survival; however, the majority $(4 / 6)$ of patients requiring retransplantation were rescued and are currently alive and well. There were no episodes of graft loss related to portal venous inflow and we have not had any cases of portal thrombosis after reconstruction (iliac vein graft or otherwise). The higher retransplantation rate and incidence of primary nonfunction in patients with PVT has been observed by others and has been attributed to the characteristically difficult intra- and postoperative course [2]. Our results are similar to those of Langnas et al. and others $\lfloor 1-3,17\rfloor$ and adds further testimony to the salvageability of high risk patients in need of liver transplantation [28, 29]. It is clear from this report that U.S. veterans are a skewed patient population when compared to the averages cited in recent reports by 
UNOS; however, this did not adversely impact upon patient survival to a significant degree $[30,31]$.

In summary, although we could not identify any significant risk factors for native PVT in our series of patients, it would appear that the group as a whole has a high prevalence of many of the risk factors alluded to in previously reported series $[1-5,17]$. Our patient population is predominantly male with a high incidence of postnecrotic cirrhosis and advanced disease (UNOS 3-4, high mean Childs-Pugh Score). Perhaps these features account for our observed high incidence of PVT in U.S. veterans undergoing OLTX. A patient, flexible approach to portal revascularization in these patients can yield quite acceptable survival and morbidity rates.

\section{REFERENCES}

1. Stieber, A. C., Zetti, G., Todo, S., Tzakis, A., Fung, J. J., Marino, I. R., Casavilla, A., and Starzl, T. E. The spectrum of portal vein thrombosis in liver transplantation. Ann. Surg. 213: 199, 1991.

2. Shaked, A., and Busuttil, R. W. Liver transplantation in patients with portal vein thrombosis and central portacaval shunts. Ann. Surg. 214(6): 696, 1991.

3. Langnas, A. T., Marujo, W. C., Stratta, R. J., Wood, R. P., Ranjan, D., Ozaki, C., and Shaw, B. W. A selective approach to preexisting portal vein thrombosis in patients undergoing liver transplantation. Ann. J. Surg. 163: 132, 1992.

4. Nonami, T., Yokoyama, I., Iwatsuki, S., and Starzl, T. E. The incidence of portal vein thrombosis at liver transplantation. Hepatology 15(5): 1195, 1991.

5. Neuhaus, P., Bechstein, W. O., Blumhardt, G., and Steffen, R. Management of portal vein thrombosis in hepatic transplant recipients. Surg. Gynecol. Obstet. 171: 251, 1990.

6. Hunt, A. H., and Wittard, B. R. Thrombosis of the portal vein in hepatic cirrhosis. Lancet 1: 218, 1954.

7. Okuda, K., Ohnishi, K., Kimura, K., Matsutani, S., Sumida, M., Goto, S., and Musha, H., et al. Incidence of portal vein thrombosis in liver cirrhosis. Gastroenterology 89: 279, 1985.

8. Belli, L., Sansalone, C. V., Aseni, P., Romani, F., and Romani, G. Portal vein thrombosis in cirrhotics. Ann. Surg. 203: 286, 1986.

9. Sarfeh, I. J. Portal vein thrombosis associated with cirrhosis. Arch. Surg. 114: 902, 1979.

10. Shiel, A. G. R., Thompson, J. F., Stevens, M. S., Eyers, A. A., Graham, J. C., and Bookallil, M. J. Mesoportal graft for thrombosed portal vein in liver transplantation. Clin. Transplant. 1: $18,1987$.

11. Shaw, B. W., Iwatsuki, S., Bron, K., and Starzl, T. E. Portal vein grafts in hepatic transplantation. Surg. Gynecol. Obstet. 1612: $66,1985$.

12. Tzakis, A., Todo, S., Stieber, A., and Starzl, T. E. Venous jump graft for liver transplantation in patients with portal vein thrombosis. Transplantation 48: 530, 1989.

13. Hiatt, J. R., Quinones-Baldrich, W. J., Ramming, K. P., Lois, J. F., and Busutill, R. W. Bile duct varices. An alternative to portoportal anastomosis in liver transplantation. Transplantation 42: 85, 1986.

14. Kirsh, J. P., Howard, T. K., Klintmalm, G. B., Husberg, B. S., and Goldstein, R. M. Problematic vascular reconstruction in liver transplantation. Portovenous conduits. Surgery 107: 544, 1990.

15. Burdick, J. F., Pitt, H. A., Colombani, P. M., Perler, B. A., Klein, A. S., Merrit, W., and Williams, G. M. Superior mesenteric inflow for liver transplantation when the portal vein is occluded. Surgery 107: 544, 1990.

16. Williams, J. W., Britt, L. G., Peters, T. G., Vera, S. R., and Haggitt, R. C. Portal vein obstruction in patients requiring hepatic resection or transplantation. Am. Surg. 50: 365, 1984.

17. Mor, E., Patel, T., Emre, S., Katz, E., Scwhartz, M., and Miller, C. Portal vein thrombosis in liver transplantation-Risk factors and results. Hepatology 16(2): 285A, 1992 [Abstract 962].

18. Mieles, L. A., Fung, J. J., Yokoyama, I., McCauley, J., Singh, N., Todo, S., Gordon, R. D., and Starzl, T. E. Liver transplantation of American veterans under FK 506 immunosuppression: a preliminary report. Trans. Proc. 23(6): 3016, 1991.

19. Starzl, T. E., Halgrimson, C. G., Koep, L. J., Weil, R., III, and Taylor, P. D. Vascular homografts from cadaveric organ donors. Surg. Gynecol. Obstet. 149: 737, 1979.

20. Starzl, T. E., Hakala, T. R., Shaw, B. W., Jr., Hardesty, R. L., Rosenthal, T. J., Griffith, B. P., Iwatsuki, I., and Bahnson, H. T. A flexible procedure for multiple cadaveric organ procurement. Surg. Gynecol. Obstet. 158: 223, 1984.

21. Lerut, J., Tzakis, A. C., Bron, K., Gordon, R. D., Iwatsuki, S., Esquivel, C. O., and Makowka, L., et al. Complications of venous reconstruction in human orthotopic liver transplantation. Ann. Surg. 205: 404, 1987.

22. Alpren, M. B., Rubin, J. M., Williams, D. M., and Capek, P. Portahepatis: Duplex doppler US with angiographic correlation. Radiology 162: 53, 1987.

23. Raby, H., Karani, J., and Powell-Jackson, P., et al. Assessment of portal vein patency: comparison of arterial portography and ultrascanning. Clin. Radiol. 39: 381, 1988.

24. Ralls, P. W. Color doppler sonography of the hepatic artery and portal venous system. Ann. J. Roentgenol. 155: 517, 1990.

25. Colonna, J. O., Winston, D. J., and Brill, J. E., et al. Infectious complication in liver transplantation. Arch. Surg. 123: 360, 1988.

26. Castaldo, P., Stratta, R. J., and Wood, P., et al. Clinical spectrum of fungal infections after orthotopic liver transplantation. Arch. Surg. 126: 149, 1991.

27. Singh, N., Gayowski, T., Wagener, M., and Yu, V. L. Infectious complications in liver recipients on Tacrolimus: Prospective analysis of 88 consecutive liver transplants. Transplantation 58(7): 744, 1994.

28. Bronsther, O., Fung, J. J., Tzakis, A., Van Thiel, D., and Starzl, T. E. Priorization and organ distribution for liver transplantation. JAMA 271: 140, 1994.

29. Eghtesad, B., Bronsther, O., Irish, W., Casavilla, A., Abu-Elmagd, K., Van Thiel, D., Tzakis, A., Fung, J. J., and Starzl, T. E. Disease gravity and urgency of need as guidelines for liver allocation. Hepatology 20(1): 56S, 1994.

30. 1993 Annual Report of the U.S. Scientific Registry for Transplant Recipients and Organ Procurement and Transplantation Network-Transplant Data: 1988-1991. UNOS, Richmond, VA, and the Division of Organ Transplantation, Bureau of Health Resources Development, HRSA, U.S. Dept. of Health and Human Services, Bethesda, MD.

31. 1991 Report of Center-Specific Graft and Patient Survival Rates (Rockville, MD). U.S. Department of Health Resources and Services, Public Health Service, Health Resources and Services Administration, Bureau of Health Resources Development, Division of Organ Transplantation (1992). 